\title{
Impact of defective interfering particles on virus replication and antiviral host response in cell culture-based influenza vaccine production
}

Timo Frensing ${ }^{1 * \ddagger}$, Antje Pflugmacher $^{1 \ddagger}$, Mandy Bachmann $^{1}$, Britta Peschel $^{1}$, Udo Reichl ${ }^{1,2}$

${ }^{1}$ Bioprocess Engineering, Max Planck Institute for Dynamics of Complex Technical Systems, Sandtorstrasse 1, 39106 Magdeburg, Germany

${ }^{2}$ Chair of Bioprocess Engineering, Otto-von-Guericke University Magdeburg, Universitaetsplatz 2, 39106 Magdeburg, Germany

${ }^{\ddagger}$ contributed equally

${ }^{*}$ corresponding author:

Jun.-Prof. Dr. Timo Frensing

Max Planck Institute for Dynamics of Complex Technical Systems

Bioprocess Engineering

Sandtorstrasse 1

39106 Magdeburg

Germany

E-mail: frensing@mpi-magdeburg.mpg.de

Phone: +493916110332

Fax: +49 3916110598 


\section{Abstract}

During the replication of influenza viruses defective interfering particles (DIPs) can be generated. These are non-infectious deletion mutants that require co-infection with a wild type virus, but interfere with its helper virus replication. Consequently, coinfected cells mainly produce DIPs. Little is known about how such non-infectious virus particles affect the virus yield of cell culture-based influenza vaccine production. We compared infections of Madin-Darby canine kidney cells with two seed virus preparations of the influenza virus strain A/Puerto Rico/8/34 that contain different amounts of DIPs. A combination of conventional RT-PCR, RT-qPCR and flow cytometry revealed that DI genomes indeed strongly accumulate in co-infected cells and impede the viral RNA synthesis. Additionally, cells infected at the higher DIP concentration showed a stronger antiviral response characterized by increased interferon- $\beta$ expression and apoptosis induction. Furthermore, in the presence of DIPs a significant fraction of cells did not show any productive accumulation of viral proteins at all. Together these effects of DIPs significantly reduce the virus yield. Therefore, the accumulation of DIPs should be avoided during influenza vaccine production which can be achieved by quality controls of working seed viruses based on conventional RT-PCR. The strategy for the depletion of DIPs presented here can help to make cell culture-based vaccine production more reliable and robust.

Keywords: Influenza virus, cell culture-based vaccine production, defective interfering particles 


\section{Introduction}

Influenza viruses cause respiratory disease and represent a continuous threat for human health. The best protection against influenza is vaccination, but due to continuous antigenic changes of the virus an annual reformulation of influenza vaccines is required. So far, most influenza vaccines are produced in embryonated chicken eggs. However, influenza vaccine production using the egg-based system is inflexible, poorly scalable and some people suffer from allergic reactions to egg components that are present in these vaccines. Additionally, each year millions of high quality fertile eggs have to be available for the vaccine production. In contrast, influenza vaccine production using cell culture overcomes these problems and hence has become an important alternative to the egg-based process (Audsley and Tannock 2008; Tree et al. 2001; Ulmer et al. 2006). The mammalian cell line MadinDarby canine kidney (MDCK) is known for its high susceptibility to different influenza strains and produces high virus yields (Gaush and Smith 1968; Merten et al. 1996). Consequently, these cells are commonly used for the production of influenza viruses (Genzel and Reichl 2009). However, vaccine virus strains used for the production are still generated in eggs (Gerdil 2003) and typically several passages in cell culture are needed to achieve an adaptation of these strains to the production cell line (Roedig et al. 2011). Vaccine virus strains are high-growth reassortants which typically contain six genome segments of a well-characterized high yield production strain, such as influenza A/Puerto Rico/8/34 (PR8), and two genome segments of the current wild type virus coding for the membrane proteins hemagglutinin and neuraminidase to provide the required immunogenicity (Fulvini et al. 2011).

In previous studies of our group different influenza A virus strains and reassortants were used to infect MDCK cells at different multiplicities of infection (MOIs) and 
infection dynamics, virus-induced apoptosis and virus yields were analyzed (Isken et al. 2012; Schulze-Horsel et al. 2009). It was found that one PR8 isolate showed an MOI dependency. Low MOIs resulted in high virus yields for infectious as well as non-infectious virus particles. In contrast, infections at high $\mathrm{MOI}$ resulted in low amounts of infectious virus particles and also reduced levels of non-infectious viruses (Isken et al. 2012). Such behavior can be caused by the presence of defective interfering particles (DIPS).

In 1951, von Magnus first showed that serial undiluted passaging of influenza virus in embryonated chicken eggs leads to a drop in virus titers (von Magnus 1951). In addition, the ratio of infectious particles to total virus particles was massively decreased because undiluted passaging produced mainly non-infectious, incomplete particles. These incomplete particles interfere with complete particles during virus propagation, the so called "von Magnus phenomenon". Afterwards, the term "defective interfering particles" was introduced by Huang \& Baltimore to describe the relevant characteristics of these non-infectious particles and to differentiate them from other non-infectious, non-interfering particles. The following definitions for DIPs were established (Huang and Baltimore 1970): DIPs are non-infectious because of deletions in their genome. Therefore, they need a helper virus with a complete genome to provide the missing viral protein(s) for replication. During such coinfections, DIPs interfere specifically with the replication of the non-defective homologous standard virus. Influenza viruses are negative-strand RNA viruses with a segmented genome. Their DIPs arise from internal deletions in one or more of the genome segments. These DI RNAs were reported to originate predominantly from the polymerase encoding genes (Nayak et al. 1985). 
In this study, we analyzed the impact of DIPs on virus replication in MDCK cells. Two different seed virus preparations were used that contain high or low amounts of DIPs. We show that DIPs not only interfere with the RNA synthesis of the standard virus but also induce a strong host cell response leading to reduction of virus yields, which can severely affect cell culture-based influenza vaccine production.

\section{Materials and Methods}

\section{Cell culture and virus infections}

Adherent MDCK cells (ECACC No.84121903) were grown in GMEM (GIBCO) supplemented with $10 \%(\mathrm{v} / \mathrm{v})$ fetal calf serum (FCS; PAN Biotech) and $1 \%(\mathrm{v} / \mathrm{v})$ peptone (Lab M) using T175 flasks and incubated at $37^{\circ} \mathrm{C}$ under a $5 \% \mathrm{CO}_{2}$ atmosphere to maintain $\mathrm{pH}$ 7.2. One day before infection cells were washed twice with phosphate buffered saline (PBS), detached and counted with a Vi-CELL ${ }^{\circledR} \mathrm{XR}$ (Beckman Coulter). Subsequently, $1.75 \times 10^{6}$ cells were seeded into $35 \mathrm{~mm}$ dishes. Infection was performed using influenza A/Puerto Rico/8/34 (National Institute for Biological Standards and Control (NIBSC) No.06/114). Prior to infection cells were washed twice with PBS and virus was added at a multiplicity of infection (MOI) of 1 in $500 \mu \mathrm{L}$ serum-free medium (GMEM, GIBCO) containing $1 \%(\mathrm{v} / \mathrm{v})$ peptone (Lab M) and $5 \mathrm{U} / \mathrm{mL}$ trypsin (GIBCO). Dishes were incubated for 30 minutes at $37^{\circ} \mathrm{C}$ and $5 \%$ $\mathrm{CO}_{2}$ atmosphere and subsequently the medium was increased to $2 \mathrm{~mL}$.

\section{Measurement of virus titer}

Total virus particle concentrations were determined using a hemagglutination assay as described previously (Kalbfuss et al. 2008). Titers were expressed as $\log _{10} \mathrm{HA}$ units per test volume (log $\mathrm{HAU} / 100 \mu \mathrm{L})$. Assuming that the HA titer corresponds to a 
virus particle concentration that equals the amount of erythrocytes used $\left(2 \times 10^{7}\right.$ cells $/ \mathrm{mL}$ ), $\log \mathrm{HAU} / 100 \mu \mathrm{L}$ can be converted into virions $/ \mathrm{mL}$ based on the equation:

$c_{\text {virus }}=2 \cdot 10^{7}$ cells $/ \mathrm{mL} \cdot 10^{(\log H A U / 100 \mu L)}$

Infectious virus particle concentrations were defined by the tissue culture infectious dose $50\left(T C I D_{50}\right)$ assay as described previously (Genzel 2007).

\section{Flow cytometric analysis}

Sample preparation and staining for flow cytometric analysis were performed as described previously (Isken et al. 2012) using one $35 \mathrm{~mm}$ dish for each time point. A fluorescein isothiocyanate (FITC)-labeled anti-NP mAb (AbD Serotec) was used to stain the viral nucleoprotein (NP) in virus-infected cells. Additionally, a TUNEL assay (Roche Diagnostics) was performed according to the manufacturer's instructions to detect apoptotic cells.

\section{Viral RNA extraction}

For the extraction of the intracellular viral RNA cells were washed once with PBS, lysed and scraped from the dish. Lysates were stored at $-80^{\circ} \mathrm{C}$. RNA was extracted using "INSTANT Virus RNA" (Analytik Jena) according to the manufacturer's instructions and stored at $-80^{\circ} \mathrm{C}$. The extraction of the viral RNA from the cell culture supernatant was done using "NucleoSpin RNA Virus" (Macherey \& Nagel) according to the manufacturer's instructions and stored at $-80^{\circ} \mathrm{C}$.

\section{Segment-specific PCR}


The reverse transcription for the subsequent amplification of all eight influenza virus genome segments by PCR was performed using the Uni12-primer (AGCAAAAGCAGG) (Hoffmann et al. 2001). $4 \mu \mathrm{L}$ of viral RNA were mixed with $1 \mu \mathrm{l}$ primer $(10 \mu \mathrm{M}), 1 \mu \mathrm{L}$ dNTPs (10 mM each) and filled up to $14 \mu \mathrm{L}$ with nuclease-free water. The mixture was incubated at $65^{\circ} \mathrm{C}$ for 5 minutes and subsequently cooled to $4{ }^{\circ} \mathrm{C}$. Afterwards, the reaction mixture $(4 \mu \mathrm{L} 5 \mathrm{X}$ Reaction Buffer, $1 \mu \mathrm{L}$ RevertAid $\mathrm{H}$ Minus Reverse Transcriptase (200 U/ $\mu \mathrm{L}$; Thermo Scientific) and $1 \mu \mathrm{L}$ nuclease-free water) was added. After incubation at $42{ }^{\circ} \mathrm{C}$ for 60 minutes the reaction was terminated at $70{ }^{\circ} \mathrm{C}$ for 10 minutes. $1 \mu \mathrm{L}$ of cDNA was mixed with $4 \mu \mathrm{L} 5 \mathrm{X}$ Phusion HF Buffer, $2 \mu \mathrm{L} \mathrm{MgCL}_{2}$ (10 mM), $1 \mu \mathrm{L}$ dNTPs (10 mM each), $1 \mu \mathrm{L}$ forward primer $(10 \mu \mathrm{M}), 1 \mu \mathrm{L}$ reverse primer $(10 \mu \mathrm{M})$ (supplementary table $\mathrm{S} 2), 0.25 \mu \mathrm{L}$ Phusion

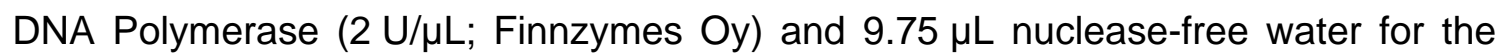
gene-specific PCR. The mixture was denaturated at $98^{\circ} \mathrm{C}$ for 3 minutes followed by 25 cycles with $98^{\circ} \mathrm{C}$ for $25 \mathrm{sec}, 54^{\circ} \mathrm{C}$ for $45 \mathrm{sec}$ and $72{ }^{\circ} \mathrm{C}$ for $1-2$ minutes (segment 1-3 $2 \mathrm{~min}, 1.5 \mathrm{~min}$ for 4-6 and $1 \mathrm{~min}$ for 7 and 8 ). The final elongation was performed at $72{ }^{\circ} \mathrm{C}$ for 10 minutes. The PCR products were directly analyzed using gel electrophoresis.

\section{In vitro synthesis of RNA reference standards}

To quantify all three RNA species from segment 5 and 7 corresponding RNA reference standards were generated by in vitro transcription. For this purpose plasmids carrying the full-length segment 5 and 7 were used as templates for PCRs in which primer sets were used to introduce a T7 promoter sequence in the desired orientation into the PCR products (see supplementary table S3). Denaturation was performed at $98{ }^{\circ} \mathrm{C}$ for 3 minutes followed by 35 cycles with $98^{\circ} \mathrm{C}$ for $25 \mathrm{sec}, 53^{\circ} \mathrm{C}$ for $45 \mathrm{sec}$ and $72{ }^{\circ} \mathrm{C}$ for $90 \mathrm{sec}$. The final elongation was at $72{ }^{\circ} \mathrm{C}$ for 10 minutes. 
The PCR products were purified using "InnuPrep PCRpure Kit" (Analytik Jena). In vitro transcription was performed using "TranscriptAid ${ }^{\mathrm{TM}}$ T7 High Yield Transcription Kit“ (Thermo Scientific) according to manufacturer's instructions. Afterwards, RNA reference standards were purified using "NucleoSpin RNA Clean-up" (Macherey \& Nagel), concentrations were determined by spectrophotometry and the RNA integrity was analyzed on a $1 \%$ formaldehyde agarose gel.

\section{Real-time RT-qPCR}

For the real-time RT-qPCR a priming strategy was used that enables the differentiation of all three viral RNA species (adapted from (Kawakami et al. 2011)). In brief, polarity specific and tagged primers (supplementary table S4) were used in reverse transcription. Therefore, $1 \mu \mathrm{L}$ of RNA extract was mixed with $1 \mu \mathrm{L}$ primer ( $1 \mu \mathrm{M}$ for cRNA and vRNA; $10 \mu \mathrm{M}$ for mRNA), $1 \mu \mathrm{L}$ dNTPs (10 mM each) and filled up to $14.5 \mu \mathrm{L}$ with nuclease-free water. The mixture was incubated at $65^{\circ} \mathrm{C}$ for 5 minutes and subsequently cooled to $42^{\circ} \mathrm{C}$ for mRNA and $55^{\circ} \mathrm{C}$ for cRNA and vRNA. Afterwards, the reaction mixture $(4 \mu \mathrm{L} 5 \mathrm{X}$ Reaction Buffer, $0.5 \mu \mathrm{L}$ Maxima H-Minus Reverse Transcriptase (200 U/ $\mu \mathrm{L}$; Thermo Scientific) and $1 \mu \mathrm{L}$ nuclease-free water) was added. After incubation at $60{ }^{\circ} \mathrm{C}$ for 30 minutes the reaction was terminated at $85^{\circ} \mathrm{C}$ for 5 minutes.

Additionally, a 10-fold dilution series of the corresponding RNA reference standards

( $5 \mathrm{ng}$ to $5 \times 10^{-7} \mathrm{ng}$ ) containing $350 \mathrm{ng}$ cellular total RNA was reverse transcribed. Subsequently, RT reactions were diluted to a final volume of $100 \mu \mathrm{L}$. Concentration of viral RNA was determined in molecules per cell using "Rotor-Gene SYBR Green PCR Kit" and Rotor-Gene Q real-time PCR cycler (Qiagen). Therefore, $4 \mu \mathrm{L}$ of the diluted cDNA were mixed with $1 \mu \mathrm{L}$ primer set (supplementary table S5) and $5 \mu \mathrm{L}$ reaction mixture. The cycle conditions of the real-time PCR were $95^{\circ} \mathrm{C}$ for 5 minutes 
followed by 40 cycles of $95^{\circ} \mathrm{C}$ for $10 \mathrm{sec}$ and $60^{\circ} \mathrm{C}$ for $20 \mathrm{sec}$. Finally, a melting curve from $65{ }^{\circ} \mathrm{C}$ to $90{ }^{\circ} \mathrm{C}$ was performed. The concentration of viral RNA was calculated based on the RNA reference standards with linear regression (Ct-value against $\log _{10}$ of number of molecules). The number of viral RNA molecules ( $n_{\text {molecules }}$ ) was calculated based on the length of the fragment $\left(\mathrm{N}_{(\text {bases }}[\mathrm{bp}]\right)$.

$n_{(\text {molecules })}=\frac{m_{\text {template }}}{k \cdot N_{(\text {bases })} \cdot N_{A}^{-1} \cdot 10^{9}}$

with the quantity of the template ( $\left.m_{\text {template }}[n g]\right)$, the average mass of one base $(k=340[\mathrm{Da} / \mathrm{bp}])$ and the Avogadro constant $\left(\mathrm{N}_{\mathrm{A}}\left[\mathrm{mol}^{-1}\right]\right)$. The number of RNA molecules was then normalized to the number of cells ( $\left.n_{\text {cells }}\right)$ to calculate numbers of

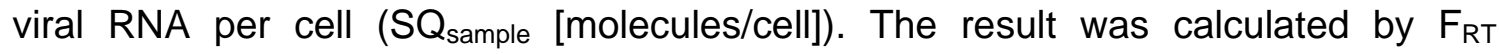
(coefficient for dilution of RT reaction) and $\mathrm{V}_{\text {eluate }}$ (volume RNA eluate $[\mu \mathrm{L}]$ ) (3).

$S Q_{\text {sample }}=\frac{10^{\left(\frac{C t-b}{m}\right)} \cdot F_{R T} \cdot V_{\text {eluate }}}{n_{\text {cells }}}$

Measurements of the interferon-beta expression were performed as described previously (Seitz et al. 2012). 


\section{Results}

In a previous study we observed that for some influenza viruses lower multiplicities of infection resulted in higher infectious virus titers (Isken et al. 2012). In particular, for a specific virus working seed of the influenza strain A/Puerto Rico/8/34 titers dropped significantly at higher MOIs. To analyze if such an MOI dependency is caused by the presence of DIPs in the working seed we aimed to produce a seed virus containing a higher proportion of infectious and lower amounts of non-infectious as well as DI particles. Therefore, MDCK cells were infected at a low MOI of 0.00025 and the supernatant was harvested at 42 hours post infection (h p.i.). Under these conditions the concentration of infectious virus particles measured by $\mathrm{TCID}_{50}$ assay could be increased to $1.29 * 10^{9}$ viruses $/ \mathrm{mL}$ compared to the starting working seed with $5.43^{\star} 10^{6}$ viruses $/ \mathrm{mL}$ (Table 1 ). Additionally, the excess of non-infectious particles of the starting seed virus material was reduced from 463:1 (non-infectious to infectious particles) to approximately 4:1. To see whether DIPs were present in both virus preparations a segment-specific RT-PCR for all eight influenza virus genome segments was performed. Therefore, eight primer sets binding to the ends of each genome segment were used separately to amplify full-length copies of all segments. This segment-specific RT-PCR also amplifies defective interfering RNAs of influenza virus DIPs as these arise from internal deletions and still contain the primer binding sites at the 5' and 3' ends. High amounts of DI RNAs were found in the starting seed virus indicated by smaller PCR products in the range between 400 and 800 bp (Fig. 1A). For the seed virus with only $5.43^{\star} 10^{6}$ infectious viruses $/ \mathrm{mL}$, DI RNAs were present for the segments 1, 2, 3 and 6 as indicated by arrows. In contrast, the new highly infectious seed virus with $1.29 * 10^{9}$ viruses $/ \mathrm{mL}$ contained predominantly the full-length genes. However, small amounts of DI RNAs were still present. Cloning and subsequent sequencing of the shorter PCR products confirmed that they contain both 
ends of an influenza virus genome segment but carry internal deletions (data not shown). Therefore, two seed virus preparations of the influenza PR8 strain were established that contained different proportions of infectious, non-infectious and DI particles. To assess the impact of the seed virus composition with respect to viral replication dynamics and virus yields, infection experiments were carried out using the starting seed virus with $5.43^{\star} 10^{6}$ viruses $/ \mathrm{mL}$ (hereafter referred to as highDIPs) and the new highly infectious seed virus with $1.29 * 10^{9}$ viruses $/ \mathrm{mL}$ (referred to as lowDIPs). For this purpose, MDCK cells were seeded in $35 \mathrm{~mm}$ dishes and infected at an MOI of 1 in a reduced volume of $500 \mu \mathrm{L}$ to achieve a synchronized infection of the cell population. With these infection conditions the $\mathrm{MOI}$ of 1 represents the highest MOI that could be used due to the low concentration of infectious viruses in the highDIPs seed stock. After 30 minutes incubation at $37{ }^{\circ} \mathrm{C}$ the medium was increased to $2 \mathrm{~mL}$. During the infection experiment samples were taken at different time points to analyze replication dynamics and virus production. The experiment was performed three times and in the following a representative experiment is shown.

\section{Comparison of virus yields}

Supernatants were taken to determine the titer of infectious and total virus particles by $\mathrm{TCID}_{50}$ and hemagglutination assay $(\mathrm{HA})$, respectively. As shown in Fig. 2A, infections with the highDIPs preparation led to higher HA titers at early time points due to the 463-fold excess of non-infectious particles. For both seed viruses the HA titers start to increase between $8 \mathrm{~h}$ p.i. and $12 \mathrm{~h}$ p.i.. However, during the infection with the lowDIPs seed virus many more virus particles were produced which can be seen from the steeper slope and higher final HA titer. At $32 \mathrm{~h}$ p.i. the HA titers of both infections showed a difference of $0.6 \log \mathrm{HAU} / 100 \mu \mathrm{L}$. Thus, a fourfold higher amount 
of total virus particles was produced during the infection with the lowDIPs seed virus. There was an even more pronounced difference for the production of infectious viruses that was about 1000 times higher for the lowDIPs seed virus (Fig. 2B). The ratio of $\mathrm{TCID}_{50}$ to $\mathrm{HA}$ titer stayed below $1 \%$ for the infection with the highDIPs virus. In contrast, approximately $65 \%$ infectious virus particles were generated by the lowDIPs seed virus at $16 \mathrm{~h}$ p.i. (Fig. 2C). Afterwards, a decrease in the ratio of infectious to total virus particles was also observed for the lowDIPs preparation. Therefore, infectious virus particles seem to be produced predominantly at early time points and at later stages of an infection a lot of non-infectious viruses are released from cells.

The comparison of different PR8 isolates and high-growth reassortants (Supplementary Table S1; also used by Isken et al. (2012)) confirmed that infections with seed virus preparations with low amounts of DIPs lead to higher virus yields (Supplementary Figure S1). Hence, for vaccine virus strains not only the nature of their HA and NA or point mutations in other segments affect the virus titer but also the presence of DIPs can have a strong impact on yields in vaccine manufacturing.

\section{Infection dynamics and apoptosis induction}

A flow cytometric analysis was performed to study infection dynamics in the cell population and virus-induced apoptosis of host cells. Cells were double stained using a TUNEL assay in combination with an immunostaining of the influenza virus nucleoprotein (NP) to determine the proportion of infected non-apoptotic and infected apoptotic cells. The proportion of infected (non-apoptotic) cells increased similarly for both infections until $8 \mathrm{~h}$ p.i. (Fig. 3A). Thereafter the infections showed clear 
differences. While for the lowDIPs seed virus almost the complete cell population showed NP accumulation, only about $80 \%$ of cells that were infected by the highDIPs virus produced NP. In contrast, the proportion of infected apoptotic cells was higher for the cell population infected with the highDIPs seed virus (Fig. 3B). This stronger apoptosis induction correlated with a much stronger interferon (IFN) response of the highDIPs-infected cell population as indicated by a 37-fold higher IFN- $\beta$ expression at $8 \mathrm{~h}$ p.i. (Fig. 3C). Hence, the seed virus containing high amounts of DIPs was unable to establish a productive infection in the complete cell population and additionally induced a stronger innate immune response as well as an accelerated cell death. The combination of these effects contributed to the lower virus yield in the presence of DIPs (Fig. 2).

\section{Dynamics of viral RNA synthesis}

To further analyze how DIPs affect the influenza A virus propagation we looked at viral transcription and replication using a RT-qPCR to measure all three influenza virus RNA species, i.e. mRNA, cRNA and vRNA. This method is unable to differentiate between DI RNAs and full-length RNAs of a particular genome segment since both contain identical terminal sequences. In addition, using primers for the junction of the 3' and 5' end of a particular DI RNA is not appropiate due to the fact that a mixture of DI RNAs was present (Fig. 1) and even for one segment a variety of DI RNAs was found by sequencing in which the position of the internal deletion differed (data not shown). For the quantification segment 5 and 7 were chosen, which encode for the nucleoprotein and matrix proteins (M1 and M2), respectively. These segments were almost free of DI RNAs (see Fig. 1) and, thus, their quantification 
enabled an unbiased investigation of the viral transcription and replication in the presence of DIPs.

The infection of MDCK cells with both seed virus preparations at an MOI of 1 and the subsequent real-time RT-qPCR resulted in a detailed time course of viral RNA synthesis. Early during the infection, an increase of viral mRNA levels was found as expected. Surprisingly, viral transcription of segment 5 and 7 was not affected by the presence of DIPs since both infections showed similar results (Fig. 4A and B). Subsequently during the replication process the complementary RNA (cRNA) is synthesized which then serves as template for the synthesis of progeny vRNA. Again, the high amount of DIPs had no obvious effect on the cRNA synthesis as minor differences in cRNA levels between both infections did not show the same trend for both segments analyzed (Fig. 4C and D). However, an apparent and reproducible effect of DIPs on the vRNA synthesis was found. In particular, infections with the highDIPs seed virus resulted in almost one order of magnitude higher vRNA starting concentrations, but vRNA synthesis was much weaker in the presence of high amounts of DIPs so that $8 \mathrm{~h}$ p.i. about one order of magnitude less vRNA had been synthesized compared to the infection with the lowDIPs seed virus (Fig. 4E and F). Hence, more vRNA was introduced into the highDIPs-infected cell population by non-infectious and/or DI particles. However, vRNA synthesis was impeded by the presence of DI RNAs. For segment 7 we detected small amounts of cRNA (0.07 molecules/cell) present at the time of infection which seems to have been caused by free viral RNAs of each RNA species that are already present in the seed virus (data not shown). Interestingly, the highDIPs seed virus contained larger amounts of free viral RNA contaminations which might be related to stronger apoptosis induction and cell death when DIPs are produced as shown in Fig. 3. 
Taken together, DI RNAs seem to have an influence on the second replication step, the synthesis of progeny vRNA, but they have only a minor impact on transcription and cRNA synthesis.

\section{Determination of DI RNAs during infection}

As described before the RT-qPCR method was not appropiate to study the accumulation of diverse DI RNAs that were present in our seed virus preparation. Hence, a conventional RT-PCR for segment 1 was performed and PCR products were analyzed via gel electrophoresis. The data demonstrate that DI RNAs of approximately $400 \mathrm{bp}$ were already detectable at time of infection in cells infected with the highDIPs seed virus (Fig. 5A). Afterwards, the concentration of the DI RNA increased constantly and only a small amount of full-length vRNA was found. In contrast, no DI RNA was detected in the lowDIPs-infected cell population at early time points and a stronger accumulation of full-length vRNA took place (Fig. 5B). However, starting at $7 \mathrm{~h}$ p.i. DI RNAs were also found in cells infected with the lowDIPs preparation. For the production of this seed virus the highDIPs working seed was used at a low MOI, whereby the DIPs were depleted but not eliminated (see also Fig. 1). Hence, if high MOI infections are performed using the lowDIPs seed virus, DIPs that are still present in this preparation will accumulate to some extent at later time points.

\section{Discussion}

Cell culture-based processes for the production of influenza vaccines have become an important alternative to the conventional vaccine production in embryonated 
chicken eggs. However, strong variations in virus yields were observed in these processes if no optimization of the $\mathrm{MOI}$ and harvest time was performed (Aggarwal et al. 2011). One aspect that was speculated to cause these variations is the presence of DIPs (Aggarwal et al. 2011; Isken et al. 2012). In this study we examined the impact of DIPs on virus replication in MDCK cells which are an important cell substrate for the propagation of influenza viruses. We chose a particular seed virus preparation of the PR8 strain that showed variations in virus titers (especially at higher MOIs) (Isken et al. 2012) and established a second seed virus containing a higher proportion of infectious virus particles and less non-infectious as well as DI particles. This enabled us to directly compare virus replication using two virus preparations of the same PR8 strain that differ remarkably in their content of DIPs.

A conventional RT-PCR for all eight influenza virus genome segments showed that various defective subgenomic RNAs were present in the working seed virus that was consequently termed highDIPs seed virus (Fig. 1A). In particular, defective RNAs were clearly detectable for the polymerase encoding segments 1, 2 and 3 as well as for segment 6 which encodes for the neuraminidase. It has previously been shown that for influenza A viruses a variety of DI or subgenomic RNAs can be found that can be derived from almost all influenza virus genome segments (Jennings et al. 1983). After one passage of this highDIPs seed virus at a low MOl of 0.00025 the amount of DI RNAs was clearly reduced while more full-length viral genomes were present.

Using both seed virus preparations in an infection experiment it was confirmed that DIPs have a strong impact on the replication of infectious virions (Fig. 2). However, under these experimental conditions not only was the infectious virus titer reduced by the presence of high amounts of DIPs but the total particle concentration (calculated 
based on the HA titer) was also approximately fourfold lower. A combination of flow cytometry, quantitative RT-PCR and conventional RT-PCR was used to study how DIPs interfere with the replication of the standard virus. Even though a conventional RT-PCR cannot provide an exact quantification and the ratio of full-length to DI RNA should not be over-interpreted since smaller products are preferentially amplified during the PCR, the comparison of both infections indicate that full-length vRNA of segment 1 is not efficiently synthesized in the presence of its corresponding DI RNA which obviously accumulated. In contrast, higher concentrations of full-length segment 1 vRNA were reached during the infection with the lowDIPs seed virus (Fig. 5). These results are in line with the hypothesis that DI RNAs have an advantage during replication compared to their full-length vRNA (Nayak et al. 1985). The realtime PCR-based analysis of the viral transcription and replication of two genome segments without significant amounts of subgenomic RNAs showed that viral mRNA and cRNA synthesis of these segments were not affected by the presence of DIPS. A decrease in VRNA alone was observed during the infection with the highDIPs seed virus (Fig. 4). This was surprising taking into account that the DI RNAs of the polymerase genes strongly interfered with the synthesis of those polymerase encoding VRNAs (Fig. 5). Therefore, the polymerase protein levels reached in the presence of DI RNAs seem to be sufficient for mRNA and cRNA synthesis, but become a limiting factor for the synthesis of vRNA. It has been reported that vRNA synthesis in particular occurs in trans by soluble polymerase complexes (Jorba et al. 2009) and such unbound polymerase complexes might be limited during the replication of DI RNAs. Clearly more research is needed to understand how DI RNAs are preferentially amplified and how they interfere with the replication of standard viruses on the molecular level. 
On the level of the infected cell population, flow cytometric analysis revealed that in the presence of DIPs a significant proportion of cells did not show productive virus replication measured by the accumulation of the viral nucleoprotein. In these cells the amount of DI RNAs may have been high enough to completely inhibit standard virus replication. Additionally, the infection with the highDIPs seed virus caused a stronger antiviral response of the cell population. A massive expression of IFN- $\beta$ was observed in the presence of DIPs (Fig. 3) which might be related to the observation that the pattern recognition receptor RIG-I preferentially binds smaller influenza virus genome segments and subgenomis RNAs leading to the IFN induction (Baum et al. 2010). The potent activation of the innate immune response by DIPs can also explain why the cell population infected with the highDIPs seed virus showed a stronger apoptosis induction (Fig. 3). It was reported before that IFN expression increases the virus-induced apoptosis of host cells (Balachandran et al. 2000). Moreover, the highDIPs seed virus might contain other non-infectious particles in addition to DIPS which could contribute to the IFN and apoptosis induction. Such non-infectious but biologically active subpopulations have been proposed (Marcus et al. 2009), however very little is known about the molecular characteristics of different non-infectious particles and how they exactly induce different biological activities.

In summary, DI RNAs potently impede the replication of the corresponding polymerase encoding full-length vRNAs and also lead to lower vRNA synthesis of other genome segments. In addition, DIPs can cause a massive antiviral response accompanied by increased apoptosis induction of host cells. Both the interference as well as the antiviral response might be the reason why a proportion of cells do not show a productive virus replication at all and, in combination with a reduced life-span of infected cells due to enhanced apoptosis induction, it is clear that DIPs can have a 
severe impact on virus yield. Therefore, the accumulation of DIPs should be avoided during vaccine production and a depletion of DIPs should be considered during the generation of the vaccine seed viruses. Typically, only egg-adapted seed viruses are provided by the WHO (Gerdil 2003) and vaccine manufacturer that use cell culturebased processes have to adapt the strain to their particular production cell line which involves several passages in the new cell system (Roedig et al. 2011). These passages during the adaptation rely on sufficient high virus concentrations to enable the selection of cell culture-adapted virus variants. In practice the virus titer is not determined in between the passages due to time-constraints for vaccine manufacture. Therefore, high MOI passaging can occur during adaptation and these conditions can also favor the accumulation of DIPs if they are present in the initial virus material or if they are generated de novo during this high $\mathrm{MOI}$ passaging. The highDIPs seed virus used here was generated by three passages of an egg-adapted PR8 variant in MDCK cells. As demonstrated in this study, it is sufficient to perform only one additional low MOI passage to produce an adapted working seed with reduced amounts of DIPs. It is also important to optimize the time point of the seed virus harvest since the infectivity commonly drops dramatically at later time points (Fig. 2C). The segment-specific RT-PCR can be used to analyze if DIPs are present in the seed virus material and provides an indication if DIP depletion has to be performed. This strategy can reduce variations in the yield of cell culture-based vaccine production and can make these processes more reliable, robust and costeffective.

\section{Acknowledgments}


We thank Frank Stefan Heldt, Tanja Laske, and Andrew Easton for critical comments on the manuscript. In addition, the authors would like to thank Stefanie Luetzner for excellent technical assistance. Mandy Bachmann is supported by a grant of the German Federal Ministry of Education and Research as part of the e:Bio initiative.

The authors declare that they have no conflict of interest.

\section{References}

Aggarwal K, Jing F, Maranga L, Liu J (2011) Bioprocess optimization for cell culture based influenza vaccine production. Vaccine 29(17):3320-8 doi:10.1016/j.vaccine.2011.01.081

Audsley JM, Tannock GA (2008) Cell-based influenza vaccines: progress to date. Drugs 68(11):1483-91 doi:68112 [pii]

Balachandran S, Roberts PC, Kipperman T, Bhalla KN, Compans RW, Archer DR, Barber GN (2000) Alpha/beta interferons potentiate virus-induced apoptosis through activation of the FADD/Caspase-8 death signaling pathway. J Virol 74(3):1513--1523

Baum A, Sachidanandam R, Garcia-Sastre A (2010) Preference of RIG-I for short viral RNA molecules in infected cells revealed by next-generation sequencing. Proc Natl Acad Sci U S A 107(37):16303-8 doi:10.1073/pnas.1005077107

Fulvini AA, Ramanunninair M, Le J, Pokorny BA, Arroyo JM, Silverman J, Devis R, Bucher D (2011) Gene constellation of influenza A virus reassortants with high growth phenotype prepared as seed candidates for vaccine production. PLoS One 6(6):e20823 doi:10.1371/journal.pone.0020823

Gaush CR, Smith TF (1968) Replication and plaque assay of influenza virus in an established line of canine kidney cells. Appl Microbiol 16(4):588-94 
Genzel Y, Reichl U (2009) Continuous cell lines as a production system for influenza vaccines. Expert Review of Vaccines 8(12):1681-92 doi:10.1586/erv.09.128

Genzel YR, Udo (2007) Vaccine production - state of the art and future needs in upstream processing. In: Pörtner R (ed) Methods in Biotechnology: Animal Cell Biotechnology - Methods and Protocols. vol 24, 2nd edn. Humana Press Inc., Totowa, NJ, pp 457-473

Gerdil C (2003) The annual production cycle for influenza vaccine. Vaccine 21(16):1776-9

Hoffmann E, Stech J, Guan Y, Webster RG, Perez DR (2001) Universal primer set for the full-length amplification of all influenza A viruses. Arch Virol 146(12):2275-89

Huang AS, Baltimore D (1970) Defective viral particles and viral disease processes. Nature 226(5243):325-7

Isken B, Genzel Y, Reichl U (2012) Productivity, apoptosis, and infection dynamics of influenza A/PR/8 strains and A/PR/8-based reassortants. Vaccine 30(35):5253-61 doi:10.1016/j.vaccine.2012.05.065

Jennings PA, Finch JT, Winter G, Robertson JS (1983) Does the higher order structure of the influenza virus ribonucleoprotein guide sequence rearrangements in influenza viral RNA? Cell 34(2):619-27

Jorba N, Coloma R, Ortin J (2009) Genetic trans-complementation establishes a new model for influenza virus RNA transcription and replication. PLoS Pathog 5(5):e1000462 doi:10.1371/journal.ppat.1000462

Kalbfuss B, Knochlein A, Krober T, Reichl U (2008) Monitoring influenza virus content in vaccine production: precise assays for the quantitation of hemagglutination and neuraminidase activity. Biologicals 36(3):145-61

Kawakami E, Watanabe T, Fujii K, Goto H, Watanabe S, Noda T, Kawaoka Y (2011) Strand-specific realtime RT-PCR for distinguishing influenza vRNA, cRNA, and mRNA. J Virol Methods 173(1):1-6 doi:S0166-0934(10)00455-6 [pii]

Marcus PI, Ngunjiri JM, Sekellick MJ (2009) Dynamics of biologically active subpopulations of influenza virus: plaque-forming, noninfectious cell-killing, and defective interfering particles. J Virol 83(16):8122-30 doi:JVI.02680-08 [pii]

Merten OW, Hannoun C, Manuguerra JC, Ventre F, Petres S (1996) Production of influenza virus in cell cultures for vaccine preparation. Adv Exp Med Biol 397:141--151

Nayak DP, Chambers TM, Akkina RK (1985) Defective-interfering (DI) RNAs of influenza viruses: origin, structure, expression, and interference. Curr Top Microbiol Immunol 114:103-51

Roedig JV, Rapp E, Hoper D, Genzel Y, Reichl U (2011) Impact of host cell line adaptation on quasispecies composition and glycosylation of influenza A virus hemagglutinin. PLoS One 6(12):e27989 doi:10.1371/journal.pone.0027989

Schulze-Horsel J, Schulze M, Agalaridis G, Genzel Y, Reichl U (2009) Infection dynamics and virusinduced apoptosis in cell culture-based influenza vaccine production-Flow cytometry and mathematical modeling. Vaccine 27(20):2712-22 doi:S0264-410X(09)00240-0 [pii]

Seitz C, Isken B, Heynisch B, Rettkowski M, Frensing T, Reichl U (2012) Trypsin promotes efficient influenza vaccine production in MDCK cells by interfering with the antiviral host response. Appl Microbiol Biot 93(2):601-611 doi:DOI 10.1007/s00253-011-3569-8

Tree JA, Richardson C, Fooks AR, Clegg JC, Looby D (2001) Comparison of large-scale mammalian cell culture systems with egg culture for the production of influenza virus $A$ vaccine strains. Vaccine 19(25-26):3444-50

Ulmer JB, Valley U, Rappuoli R (2006) Vaccine manufacturing: challenges and solutions. Nat Biotechnol 24(11):1377-83 doi:10.1038/nbt1261

von Magnus P (1951) Propagation of the PR8 strain of influenza A virus in chick embryos. II. The formation of incomplete virus following inoculation of large doses of seed virus. Acta pathologica et microbiologica Scandinavica 28(3):278-93 
Fig. 1 Amplification of viral full-length and DI RNAs A segment-specific RT-PCR was performed for all eight influenza genome segments from two different influenza A/PR/8/34 seed viruses. (A) starting seed virus $\left(5.43^{\star} 10^{6}\right.$ virions $\left./ \mathrm{mL}\right)$; (B) new highly infectious seed virus $\left(1.29 * 10^{9}\right.$ virions $\left./ \mathrm{mL}\right)$; Lane 1 to 8 represent the influenza virus genome segments 1 to 8, respectively. Arrows indicate the position of DI RNAs. M: GeneRuler DNA Ladder Mix

Fig. 2 Infectious and total virus yield MDCK cells were infected with the highDIPs and lowDIPs PR8 seed virus at an $\mathrm{MOI}$ of 1 . Virus titers were determined by the HA $(A)$ and $\mathrm{TCID}_{50}(\mathrm{~B})$ assay. (C) The ratio of infectious $\left(\mathrm{TCID}_{50}\right)$ to total virus particles (HA titer) is shown.

Fig. 3 Impact of DIPs on the host cell population A flow cytometric analysis was performed to determine the amount of infected non-apoptotic cells $(A)$ and infected apoptotic cells (B) after an infection of MDCK cells with both seed virus preparations at an MOI of 1 . (C) IFN- $\beta$ expression was measured at $8 \mathrm{~h}$ p.i. using RT-qPCR.

Fig. 4 Effect of DIPs on viral transcription and replication A detailed analysis of the synthesis of viral mRNA $(A, B), \operatorname{cRNA}(C, D)$ and vRNA $(E, F)$ of segment 5 and 7 was performed for early time points after the infection of MDCK cells with both virus preparations using a real-time RT-qPCR. 
Fig. 5 Accumulation of full-length and DI RNA of segment 1. Samples of virus infected MDCK cells were taken at indicated time points and the intracellular accumulation of segment 1 was analyzed by a segment-specific conventional RT-PCR. Cells were infected with the highDIPs seed virus $(A)$ or with the lowDIPs preparation (B). Full-length (FL) segment 1 has $2341 \mathrm{bp}$ and DI RNAs were found with approximately $400 \mathrm{bp}$. 
Fig. 1
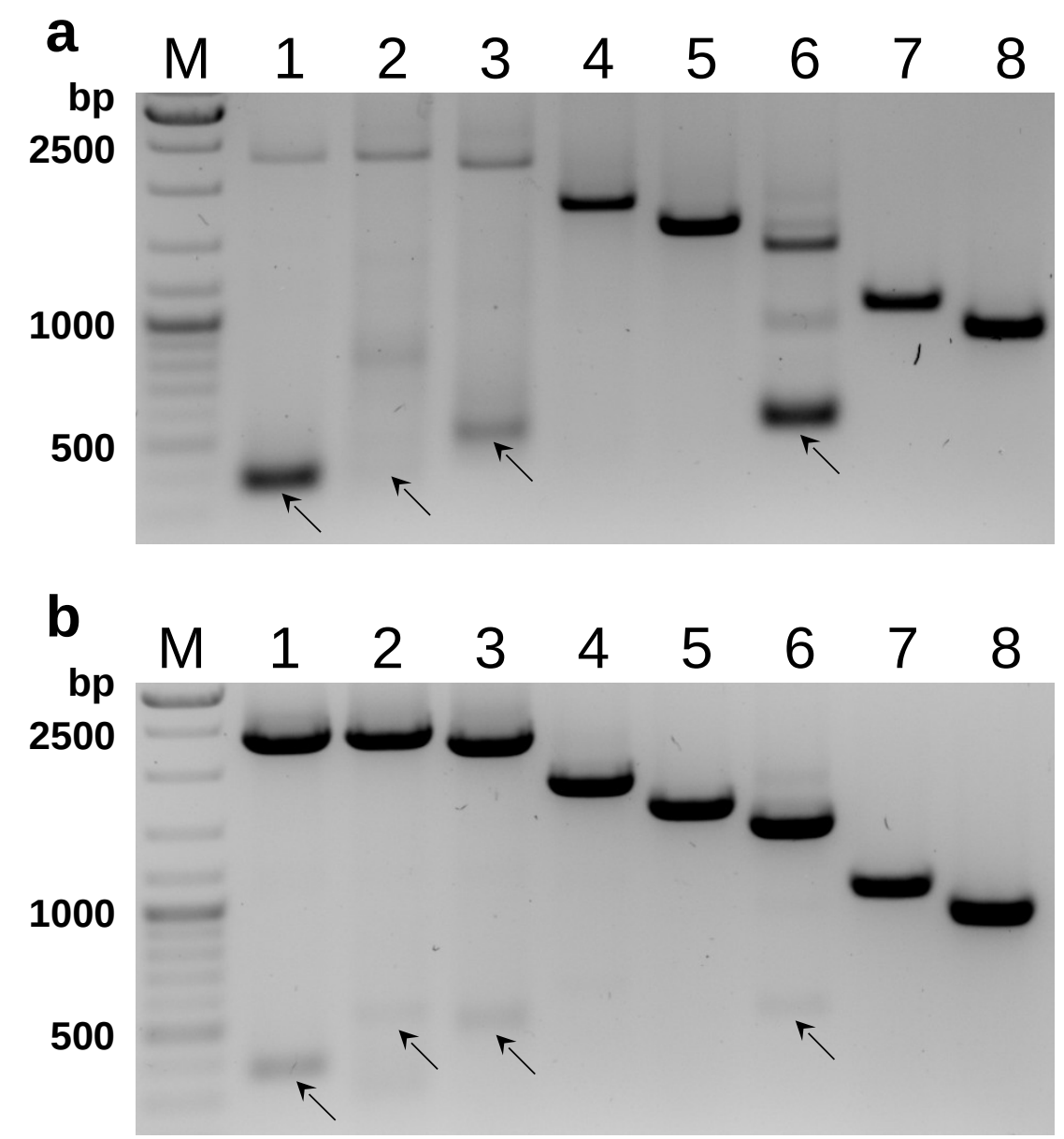
Fig. 2
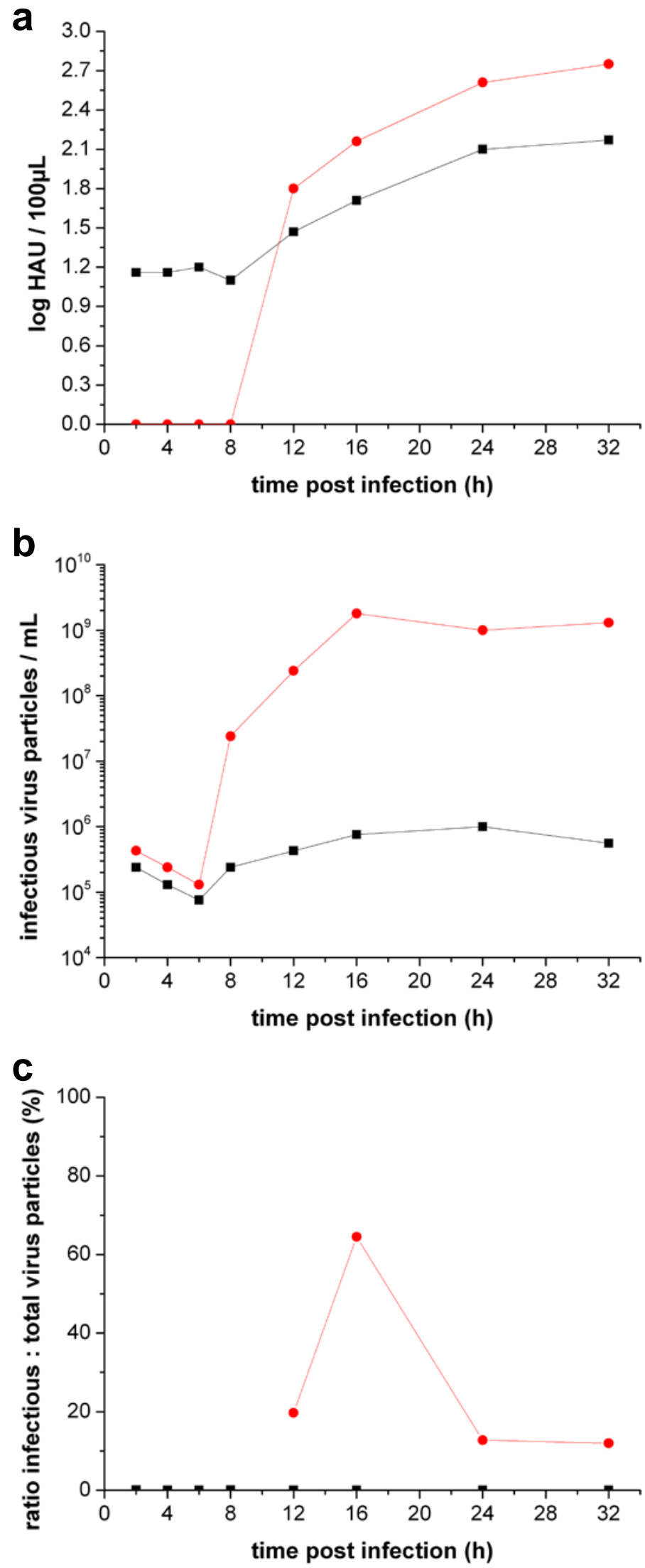

- highDIPs - lowDIPs 


$$
\text { I }
$$


Fig. 4

Segment 5
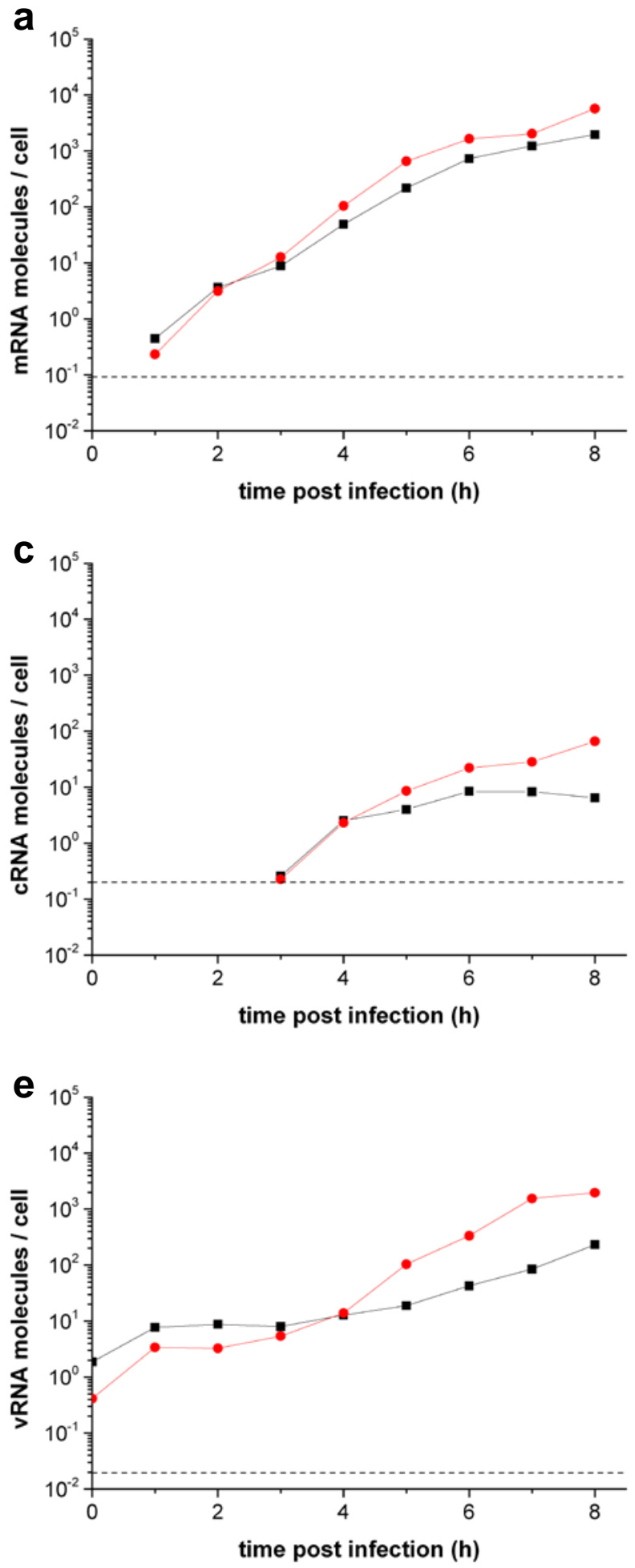

Segment 7
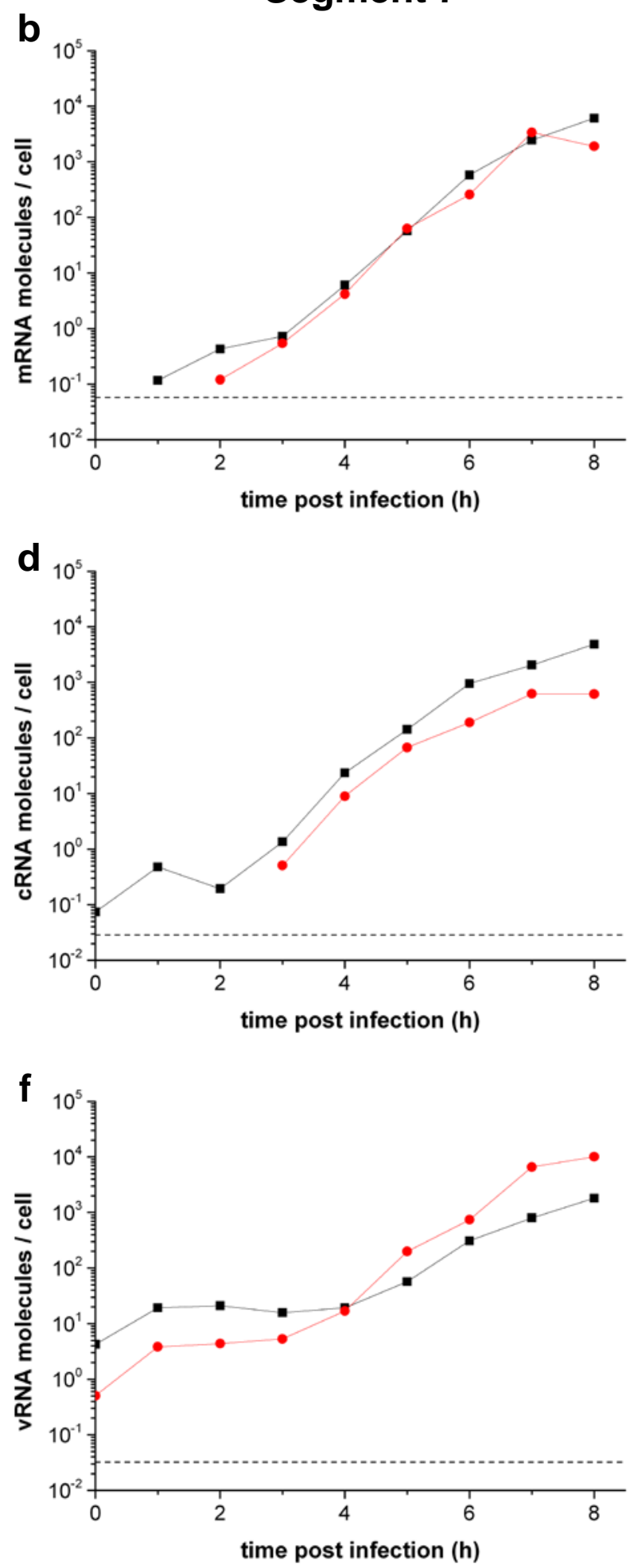

- highDIPs - lowDIPs ---- detection limit 
Fig. 5

$\begin{array}{lllllllllll}\mathbf{a} & 0 & 1 & 2 & 3 & 4 & 5 & 6 & 7 & 8 & \text { hpi } \\ & & & & & & & & & & \end{array}$

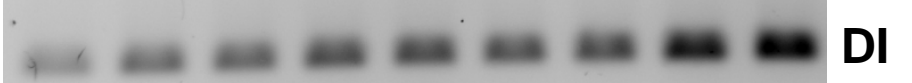

$\begin{array}{lllllllllll}\text { b } & 0 & 1 & 2 & 3 & 4 & 5 & 6 & 7 & 8 & \mathrm{hpi} \\ \mathrm{FL}\end{array}$

DI 


\section{Impact of defective interfering particles on virus replication and antiviral host response in cell culture-based influenza vaccine production}

Applied Microbiology and Biotechnology

Timo Frensing ${ }^{\star}$, Antje Pflugmacher, Mandy Bachmann, Britta Peschel, Udo Reichl

"Bioprocess Engineering, Max Planck Institute for Dynamics of Complex Technical Systems,

Sandtorstrasse 1, 39106 Magdeburg, Germany; E-mail: frensing@mpi-magdeburg.mpg.de

Supplementary table S1: Comparison of influenza virus seed preparations

\begin{tabular}{|c|c|c|c|}
\hline $\begin{array}{c}\text { Seed virus } \\
\text { preparation }\end{array}$ & $\begin{array}{c}\mathrm{TCID}_{50} \\
\text { (virions } / \mathrm{mL})\end{array}$ & $\begin{array}{c}\text { Total virus } \\
\text { particles }\end{array}$ & $\begin{array}{c}\text { Infectivity } \\
\text { (infectious:non- } \\
\text { infectious particles) }\end{array}$ \\
\hline highDIPs* $^{*}$ & $0.05 \times 10^{8}$ & $2.5 \times 10^{9}$ & $0.2 \%$ \\
\hline lowDIPs $^{*}$ & $13 \times 10^{8}$ & $5.5 \times 10^{9}$ & $23.4 \%$ \\
\hline PR8-RKI $^{\#}$ & $0.4 \times 10^{8}$ & $4 \times 10^{9}$ & $1 \%$ \\
\hline Wisconsin-like $^{\S}$ & $2.3 \times 10^{8}$ & $5.1 \times 10^{9}$ & $4.4 \%$ \\
\hline Uruguay-like $^{\ddagger}$ & $1 \times 10^{8}$ & $5.6 \times 10^{9}$ & $1.7 \%$ \\
\hline
\end{tabular}

* Generated from influenza virus A/Puerto Rico/8/34 (H1N1) from NIBSC (No.06/114)

\# Influenza virus A/Puerto Rico/8/34 (H1N1) from Robert Koch Institute (RKI)

${ }^{\S}$ High-growth reassortant NYMC X-161b (A/Wisconsin/67/2005 (H3N2), NIBSC, No. 06/242)

${ }^{\ddagger}$ High-growth reassortant NYMC X-175c (A/Uruguay/716/2007 (H3N2), NIBSC, No. 08/304)
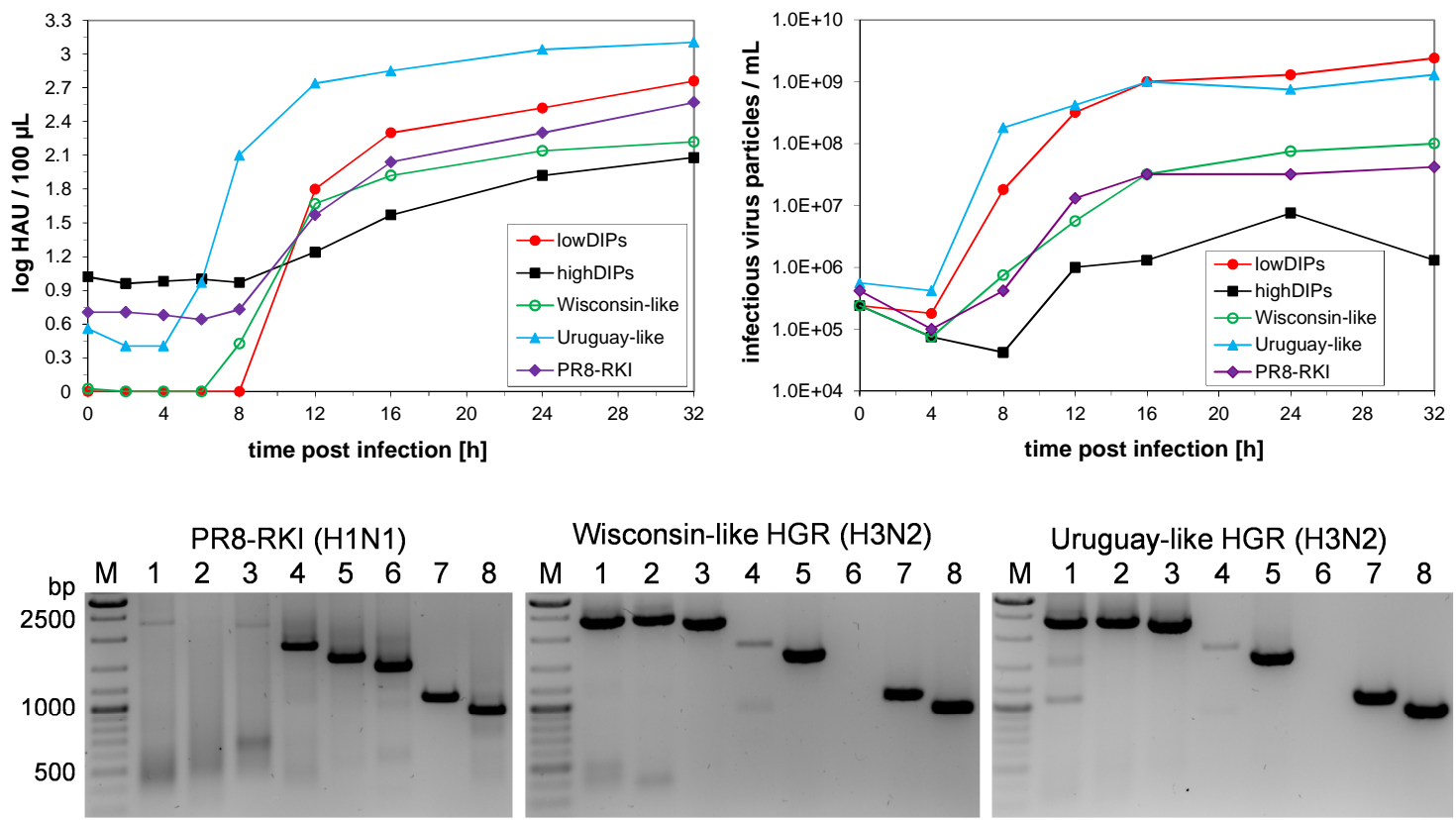

Wisconsin-like HGR (H3N2)

Uruguay-like HGR (H3N2)

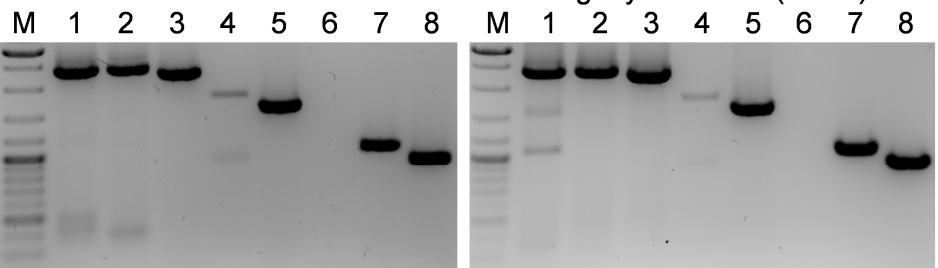

Supplementary figure S1: MDCK cells were infected at an $\mathrm{MOI}$ of 1 and virus titers were determined by HA and TCID 50 assay. A segment-specific RT-PCR was performed for all eight PR8 genome segments from the indicated PR8 isolate and high-growth reassortants. 
Supplementary table S2: Primers used for the segment-specific PCR

\begin{tabular}{llll}
\hline Target & Primer name & Sequence (5' -3') $^{\prime}$ & Position (nt) \\
\hline Segment 1 & Seg 1 Uni for & AGCGAAGCAGGTCAATTAT & $1-20$ \\
& Seg 1 Uni rev & AGTAGAAACAAGGTCGTTTTTAAAC & $2316-2341$ \\
Segment 2 & Seg 2 Uni for & AGCGAAAGCAGGCAAACCAT & $1-20$ \\
& Seg 2 Uni rev & AGTAGGAACAAGGCATTTTTTCATG & $2316-2341$ \\
Segment 3 & Seg 3 Uni for & AGCGAAAGCAGGTACTGATCC & $1-21$ \\
& Seg 3 Uni rev & AGTAGAAACAAGGTACTTTTTTGG & $2209-2233$ \\
Segment 4 & Seg 4 Uni for & AGCAAAAGCAGGGGAA & $1-16$ \\
& Seg 4 Uni rev & AGTAGAAACAAGGGTGTTTT & $1755-1775$ \\
Segment 5 & Seg 5 Uni for & AGCAAAAGCAGGGTAGATAATC & $1-22$ \\
& Seg 5 Uni rev & AGTAGAAACAAGGGTATTTTTC & $1543-1565$ \\
Segment 6 & Seg 6 Uni for & AGCGAAAGCAGGGGTTTAAAATG & $1-23$ \\
& Seg 6 Uni rev & AGTAGAAACAAGGAGTTTTTTGAAC & $1389-1413$ \\
Segment 7 & Seg 7 Uni for & AGCGAAAGCAGGTAGATATTG & $1-21$ \\
& Seg 7 Uni rev & AGTAGAAACAAGGTAGTTTTTTAC & $1004-1027$ \\
Segment 8 & Seg 8 Uni for & AGAAAAAGCAGGGTGACAAA & $1-20$ \\
& Seg 8 Uni rev & AGTAGAAACAAGGGTGTTTT & $870-890$ \\
\hline
\end{tabular}


Supplementary table S3: Primer sets for RNA reference standard generation

\begin{tabular}{|c|c|c|c|c|}
\hline Target & RNA-type & Primer name & Sequence $\left(5^{\prime}-3^{\prime}\right)$ & Position (nT) \\
\hline \multirow[t]{6}{*}{ Segment 5} & cRNA & Seg 5 Uni T7 for & TAATACGACTCACTATAGGGAGCAAAAGCAGGGTAGATAATC & $1-22$ \\
\hline & & Seg 5 Uni rev & AGTAGAAACAAGGGTATTTTTC & $1543-1565$ \\
\hline & VRNA & Seg 5 Uni for & AGCAAAAGCAGGGTAGATAATC & $1-22$ \\
\hline & & Seg 5 Uni T7 rev & TAATACGACTCACTATAGGGAGTAGAAACAAGGGTATTTTTC & $1543-1565$ \\
\hline & mRNA & Seg 5 Uni T7 for & TAATACGACTCACTATAGGGAGCAAAAGCAGGGTAGATAATC & $1-22$ \\
\hline & & Seg $5 \mathrm{dT}$ rev & TTTTTTTTTTTTTTTTTCTTTAATTGTC & $1533-1549$ \\
\hline \multirow[t]{6}{*}{ Segment 7} & cRNA & Seg 7 Uni T7 for & TAATACGACTCACTATAGGGAAGCGAAAGCAGGTAG & $1-15$ \\
\hline & & Seg 7 Uni rev & AGTAGAAACAAGGTAGTTTTT & $1006-1027$ \\
\hline & VRNA & Seg 7 Uni for & AGCGAAAGCAGGTAG & $1-15$ \\
\hline & & Seg 7 Uni T7 rev & TAATACGACTCACTATAGGGAAGTAGAAACAAGGTAGTTTTT & $1006-1027$ \\
\hline & mRNA & Seg 7 Uni T7 for & TAATACGACTCACTATAGGGAAGCGAAAGCAGGTAG & $1-15$ \\
\hline & & Seg $7 \mathrm{dT}$ rev & TTTTTTTTTTTTTTTTTACTCCAGСTCT & $995-1012$ \\
\hline
\end{tabular}


Supplementary table S4: Primers used for the reverse transcription of specific influenza virus RNA species.

\begin{tabular}{|c|c|c|c|c|}
\hline Target & RNA-type & Primer name & Sequence $\left(5^{\prime}-3^{\prime}\right)$ & Position (nt) \\
\hline \multirow[t]{2}{*}{ Segment 5} & cRNA & Seg 5 tagRT rev & GCTAGCTTCAGCTAGGCATCAGTAGAAACAAGGGTATTTTTCTT & $1541-1565$ \\
\hline & vRNA & Seg 5 tagRT for & ATTTAGGTGACACTATAGAAGCGAGTGATTATGAGGGACGGTTGAT & $192-215$ \\
\hline \multirow[t]{2}{*}{ Segment 7} & cRNA & Seg 7 tagRT rev & GCTAGCTTCAGCTAGGCATCAGTAGAAACAAGGTAGTTTTTTAC & $1003-1027$ \\
\hline & vRNA & Seg 7 tagRT for & ATTTAGGTGACACTATAGAAGCGAGCCGAGATCGCACAGAGACTT & $87-109$ \\
\hline Segment $5 \& 7$ & mRNA & Oligo tagdTRT & GTAAAACGACGGCCAGTTTTTTTTTTTTTTTTTTT & \\
\hline
\end{tabular}

Supplementary table S5: Primers used for real-time RT-qPCR

\begin{tabular}{|c|c|c|c|c|}
\hline Target & & Primer name & Sequence $\left(5^{\prime}-3^{\prime}\right)$ & Position (nt) \\
\hline Introduced & vRNA & vRNA tagRealtime for & ATTTAGGTGACACTATAGAAGCG & - \\
\hline \multirow[t]{2}{*}{ Tag sequence } & cRNA & cRNA tagRealtime rev & GCTAGCTTCAGCTAGGCATC & - \\
\hline & mRNA & mRNA tagRealtime rev & GTAAAACGACGGCCAGT & - \\
\hline \multirow[t]{2}{*}{ Segment 5} & vRNA & Seg 5 Realtime rev & CGCACTGGGATGTTCTTC & $282-300$ \\
\hline & $\mathrm{c} / \mathrm{mRNA}$ & Seg 5 Realtime for & GGAAAGTGCAAGACCAGAAGAT & $1388-1410$ \\
\hline \multirow[t]{2}{*}{ Segment 7} & vRNA & Seg 7 Realtime rev & TGAGCGTGAACACAAATCCTAAAA & $201-225$ \\
\hline & $\mathrm{c} / \mathrm{mRNA}$ & Seg 7 Realtime for & CATTGGGATCTTGCACTTGACATT & $809-831$ \\
\hline
\end{tabular}

